\title{
SOME CONSIDERATIONS ON THE NONABELIAN TENSOR SQUARE OF CRYSTALLOGRAPHIC GROUPS
}

\author{
AHMAD ERFANIAN, FRANCESCO G. RUSSO, AND NOR HANIZA SARMIN
}

\begin{abstract}
The nonabelian tensor square $G \otimes G$ of a polycyclic group $G$ is a polycyclic group and its structure arouses interest in many contexts. The same assertion is still true for wider classes of solvable groups. This motivated us to work on two levels in the present paper: on a hand, we investigate the growth of the Hirsch length of $G \otimes G$ by looking at that of $G$, on another hand, we study the nonabelian tensor product of pro- $p$-groups of finite coclass, which are a remarkable class of solvable groups without center, and then we do considerations on their Hirsch length. Among other results, restrictions on the Schur multiplier will be discussed.
\end{abstract}

\section{INTRODUCTION}

The nonabelian tensor square $G \otimes G$ of a group $G$ is the group generated by the symbols $x \otimes y$ and subject to the relations

$$
x y \otimes z=\left({ }^{x} y \otimes{ }^{x} z\right)(x \otimes z) \text { and } x \otimes z t=(x \otimes z)\left({ }^{z} x \otimes{ }^{z} t\right)
$$

for all $x, y, z, t \in G$, where $G$ acts on itself via conjugation ${ }^{x} y=x y x^{-1}$. In particular, if $G$ is abelian and acts trivially on itself, we have the usual abelian tensor product $G \otimes_{\mathbb{Z}} G$. The group $G \wedge G=G \otimes G / \nabla(G)$ is called nonabelian exterior square of $G$, where $\nabla(G)=\langle x \otimes x \mid x \in G\rangle$. From [5] the maps $\kappa: x \otimes y \in G \otimes G \longmapsto[x, y] \in[G, G]$ and $\kappa^{\prime}: x \wedge y \in G \wedge G \longmapsto[x, y] \in[G, G]$ are epimorphisms and the topological meaning of $\operatorname{ker} \kappa=J_{2}(G)$ is described in [6]. Still from [5] the following diagram is commutative with exact rows and central extensions as columns:

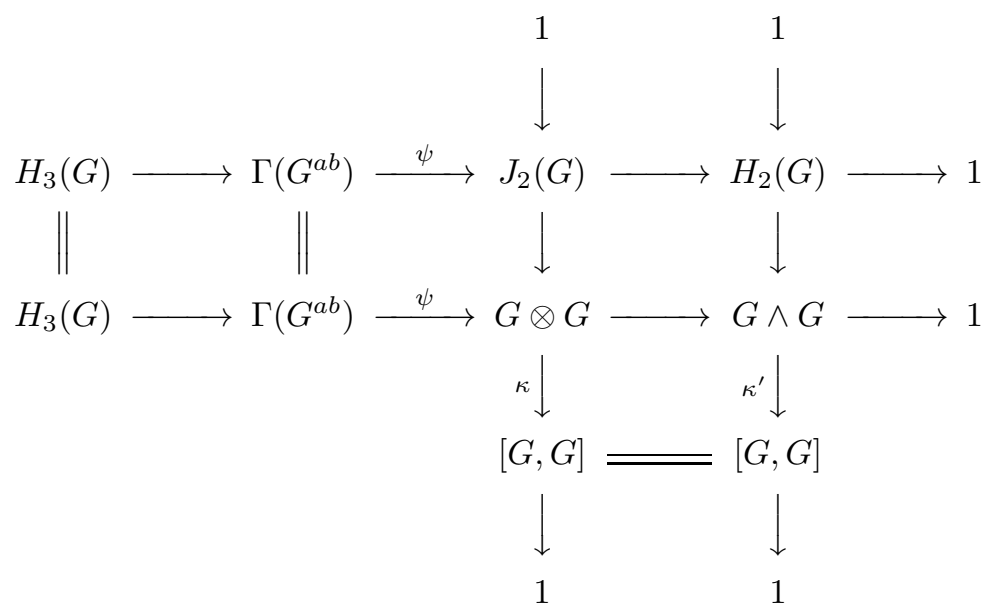

where $H_{2}(G)=\operatorname{ker} \kappa^{\prime}$ is the second integral homology group of $G, H_{3}(G)$ is the third integral homology group of $G$ and $\Gamma$ is the Whitehead's quadratic functor in [5, Section 2]. We note that $\mathrm{H}_{2}(G)$ is exactly the Schur multiplier $M(G)$ of $G$.

After the initial work [7, many authors investigated the structure of $G \otimes G$ by looking at that of $G$ and in the last times there is a significant production which is devoted to the classes $\mathfrak{P}$ of all polycyclic groups, $\mathfrak{F}$ of all finite groups and $\mathfrak{S}$ of all solvable groups (see [3, 4, 8, 16, 21]). In a solvable group $G$ we recall that the number of infinite cyclic factors $h(G)$ is an invariant, called

Date: November 13, 2018.

Key words and phrases. Hirsch length, Schur multiplier, crystallographic groups, pro- $p$-groups of finite coclass, Bieberbach groups.

Mathematics Subject Classification 2010: 20J99, 20F18. 
A. ERFANIAN, F. G. RUSSO, AND N.H. SARMIN

Hirsch length, or torsion-free rank, of $G$ (see [14, pp.14, $15,16,85]$ ). If $G \in \mathfrak{P}$, we have $h(G)=0$ if and only if $G \in \mathfrak{P} \cap \mathfrak{F}$. Now, if $G$ is abelian, then $G \otimes G$ is abelian by [21, Theorem 3.1]; if $G \in \mathfrak{P}$, then $G \otimes G \in \mathfrak{P}$ (see [4, 8, 16]) and, so far as we know, the structure of $G \otimes G$ is widely described in terms of the upper central series of $G$. For instance, [12 classifies $G \otimes G$, when $G$ is a 2-generator 2-group, and so, $G$ is a particular type of polycyclic group with nontrivial center. 19] describes $G \otimes G$, where $G$ is an infinite nonabelian 2-generator nilpotent group of class 2, and so, $G$ is still a polycyclic group with nontrivial center. There are several contributions on this line of research but it is hard to find information on $G \otimes G$ when $G$ is a polycyclic group with trivial center: we found the initial idea in $[2$ and a recent interest in $[3,8,2,15]$.

The aim of the present work is to detect the structure of $G \otimes G$, when $G$ is a polycyclic group with trivial center, or more generally an infinite solvable group with trivial center, starting from bounds on $h(G \otimes G)$ and $h(G)$. The absence of literature on such a line of investigation has motivated us to write the present paper. On another hand, R. F. Morse has kindly pointed out (see [17]) that the same question was posed by C. Rover at the Conference on Computational Group Theory and Cohomology at the Harlaxton College (Harlaxton Lincolnshire, UK) in 2008. We end this introduction, noting that the terminology and the notations of the present paper are standard and can be found in [5, 6, 7, 11].

\section{The growth of the Hirsch length in the nonabelian tensor square}

The following (unpublished) lemma was communicated to us by D. Ramras and describes some classical situations, which we may encounter, when we deal with the presentations of polycyclic groups. Further details can be found in [18.

Lemma 2.1. Let $l, p, k, m, n_{1}, n_{2}, \ldots, n_{m}$ be integers. Consider an extension of groups $1 \rightarrow A \longmapsto$ $\Gamma \rightarrow Q \rightarrow 1$ in which $A$ is a finitely generated abelian group and $Q$ is finite. If

$$
Q=\left\langle q_{1}, \ldots, q_{l} \mid r_{1}\left(q_{1}, \ldots, q_{l}\right)=\ldots=r_{p}\left(q_{1}, \ldots, q_{l}\right)=1\right\rangle
$$

for some words $r_{1}, \ldots, r_{p}$ in the free group on l letters and

$A=\left\langle a_{1}, \ldots, a_{k+m} \mid\left[a_{i}, a_{j}\right]=1(1 \leq i \leq j \leq k+m), a_{1}^{n_{1}}=\ldots=a_{m}^{n_{m}}=1\left(1 \leq n_{1} \leq \ldots \leq n_{m}\right)\right\rangle$,

then for some words $w_{i}$ and $u_{i j}$ (not uniquely determined) in the free group on $k+m$ letters,

$$
\begin{gathered}
\Gamma=\left\langle\alpha_{1}, \ldots, \alpha_{k+m}, \gamma_{1}, \ldots, \gamma_{l}\right| r_{1}\left(\gamma_{1}, \ldots, \gamma_{l}\right)=w_{1}\left(\alpha_{1}, \ldots, \alpha_{k+m}\right), \ldots, \\
r_{p}\left(\gamma_{1}, \ldots, \gamma_{l}\right)=w_{p}\left(\alpha_{1}, \ldots, \alpha_{k+m}\right),\left[\alpha_{i}, \alpha_{j}\right]=1, \alpha_{j}^{n_{j}}=1, \gamma_{i} \alpha_{j} \gamma_{i}^{-1}=u_{i j}\left(\alpha_{1}, \ldots, \alpha_{k+m}\right), \\
(1 \leq i \leq j \leq k+m)\rangle .
\end{gathered}
$$

Proof. To begin, we must specify the words $u_{i j}$ and $w_{i}$. Choose elements $\widetilde{q}_{i} \in \Gamma$ lying over $q_{i} \in Q$. Since $A$ is normal in $\Gamma$, we know that $\widetilde{q}_{i} a_{j} \widetilde{q}_{i}^{-1} \in A$, and hence $\widetilde{q}_{i} a_{j} \widetilde{q}_{i}^{-1}=u_{i j}\left(a_{1}, \ldots a_{k+m}\right)$ for some word $u_{i j}$. Next, since $r_{i}\left(q_{1}, \ldots, q_{l}\right)=1$ in $Q$, we know that $r_{i}\left(\widetilde{q}_{1}, \ldots, \ldots q_{l}\right) \in A$, and hence $r_{i}\left(q_{1}, \ldots, q_{l}\right)=w_{i}\left(a_{1}, \ldots, a_{k+m}\right)$ for some word $w_{i}$. Now, let $\widetilde{\Gamma}$ denote the group presented by (2.3)-(2.5), and let $\widetilde{A}$ denote the subgroup generated by $\alpha_{1}, \ldots, \alpha_{k+m}$. Let $\Phi: \widetilde{\Gamma} \rightarrow \Gamma$ be the homomorphism defined by $\Phi\left(\alpha_{i}\right)=a_{i}$ and $\Phi\left(\gamma_{i}\right)=\widetilde{q}_{i}$. Then $\Phi$ is surjective, and its restriction to $\widetilde{A}$ is a surjection onto $A \leq \Gamma$. The third set of relations in (2.3)-(2.5) ensures that $\widetilde{A}$ is normal in $\widetilde{\Gamma}$, and we define $\widetilde{Q}=\widetilde{\Gamma} / \widetilde{A}$. The map $\Phi$ induces a surjection $\widetilde{\Phi}: \widetilde{Q} \rightarrow Q$, and we have a commutative diagram

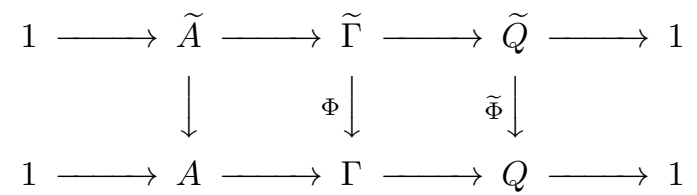

The map $\widetilde{\Gamma} \rightarrow \widetilde{Q}$ induces a surjection from the free group on the generators $\gamma_{i}$ onto $\widetilde{Q}$, and this surjection factors through the quotient group $\left\langle\gamma_{1}, \ldots \gamma_{l} \mid r_{i}\left(\gamma_{1}, \ldots, \gamma_{l}\right)=1\right\rangle \simeq Q$. Hence we have a surjection $Q \rightarrow \widetilde{Q}$, meaning that $\widetilde{Q}$ is a finite group of order at most $|Q|$. The existence of 
the surjection $\widetilde{\Phi}: \widetilde{Q} \rightarrow Q$ now shows that both of these surjections must in fact be isomorphisms. Next, we show that the map $\widetilde{A} \rightarrow A$ is injective. Each element $\alpha \in \widetilde{A}$ has the form $\alpha_{1}^{p_{1}} \alpha_{2}^{p_{2}} \ldots \alpha_{k+m}^{p_{k+m}}$ for some integers $p_{i}$. Our presentation for $A$ shows that, if $\Phi(\alpha)=0$, then $p_{i}$ is a multiple of $n_{i}$ for $1 \leq i \leq m$, and $p_{i}=0$ for $i>m$. But such elements are already trivial in $\widetilde{\Gamma}$, so $\phi$ is injective when restricted to $\widetilde{A}$. We have now shown that the two outer maps in (2.6) are isomorphisms, and the 5-lemma shows that $\Phi$ is an isomorphism as well.

Lemma 2.1 can be specialized in various ways. For instance, assume that the cyclic group $C_{n}=\left\langle t \mid t^{n}=1\right\rangle$ of order $n>1$ is equal to $Q ;$ the free abelian group $\mathbb{Z}^{n-1}=\underbrace{\mathbb{Z} \times \ldots \times \mathbb{Z}}_{(n-1) \text {-times }}=$ $\left\langle a_{1}, \ldots, a_{n-1} \mid\left[a_{i}, a_{j}\right]=1 ; 1 \leq i, j \leq n-1\right\rangle$ of rank $n-1$ is equal to $A ; C_{n}$ acts on $\mathbb{Z}^{n-1}$ via the following homomorphism

$$
\xi: t \in C_{n} \mapsto \xi(t)=\left(\begin{array}{ccccc}
0 & 1 & 0 \ldots & 0 & 0 \\
0 & 0 & 1 \ldots & 0 & 0 \\
\ldots & \ldots & \ldots & \ldots & \ldots \\
0 & 0 & 0 \ldots & 0 & 1 \\
-1 & -1 & -1 \ldots & -1 & -1
\end{array}\right) \in G L_{n-1}(\mathbb{Z}) .
$$

We have the crystallographic group $G_{n}=C_{n} \ltimes \mathbb{Z}^{n-1}$ of holonomy $n$ and several information on it can be found in [8, §6.3], or [1, Proposition 3.3]. Looking at its construction, $G_{n} \in \mathfrak{P}$, $h\left(G_{n}\right)=n-1, Z\left(G_{n}\right)=\{1\}$ and $G_{n}$ is metabelian (in particular, $\left[G_{n}, G_{n}\right]$ is abelian). On another hand, we may get a presentation for $G_{n}$, taking a generating set for $C_{n}$, another for $\mathbb{Z}^{n-1}$ and considering the action (2.7). We have as follows.

Corollary 2.2. $G_{n}=\left\langle a_{1}, \ldots, a_{n-1}, t\right| t^{n}=1, t^{-1} a_{i} t=a_{i+1}(1 \leq i \leq n-2), t^{-1} a_{n-1} t=$ $\left.a_{1}^{-1} \ldots a_{n-1}^{-1},\left[a_{i}, a_{j}\right]=1(1 \leq j<i \leq n-1)\right\rangle$.

We can be more accurate in the description of $\left[G_{n}, G_{n}\right]$ and of the abelianization $G_{n}^{a b}=$ $G_{n} /\left[G_{n}, G_{n}\right]$. In fact $\left[G_{n}, G_{n}\right]$ is generated by the commutators of generators of $G_{n}$ and their inverses. Since all the $a_{i}$ commute and $t$ has finite order, one has only to consider commutators of the form $\left[t, a_{i}\right]$ and thus

$$
\begin{gathered}
{\left[G_{n}, G_{n}\right]=\left\langle a_{i}^{-1} a_{i+1}, a_{1}^{-1} \ldots a_{n-2}^{-1} a_{n-1}^{-2}\right|} \\
\left.\left[a_{i}^{-1} a_{i+1}, a_{j}^{-1} a_{j+1}\right]=\left[a_{i}^{-1} a_{i+1}, a_{1}^{-1} \ldots a_{n-2}^{-1} a_{n-1}^{-2}\right]=1(1 \leq i, j \leq n-2)\right\rangle .
\end{gathered}
$$

We note that $\left[G_{n}, G_{n}\right]$ is free abelian of rank $n-1$. On another hand, when we factor $G_{n}$ through $\left[G_{n}, G_{n}\right]$, we have that $t$ is an independent generator and $a_{1}=a_{2}=\ldots=a_{n-1}$. So $a_{n-1}=$ $a_{n-1}^{-(n-1)}$ which implies $a_{n-1}^{n}=1$ and $a_{n-1}$ is a second independent generator. We conclude that $G_{n}^{a b}=C_{n} \times C_{n}$.

On another hand, if $G_{n}$ has $n=p^{s}\left(p\right.$ prime and $s \geq 1$ ) and we replace $\mathbb{Z}^{p-1}$ with $\mathbb{Z}_{p}^{d_{s}}$, where $\mathbb{Z}_{p}$ denotes the group of $p$-adic integers and $d_{s}=p^{s-1}(p-1)$, then we have the pro-p-group $K_{s}=C_{p^{s}} \ltimes \mathbb{Z}_{p}^{d_{s}}$ of finite coclass with central exponent $s$, studied in [9, 13. This time we cannot apply Lemma 2.1 but computational arguments are still true. We recall a result in this direction, to convenience of the reader.

Lemma 2.3 (See 9], Theorem 7). For an integer $i$ let $e_{i}=1$, if $p^{s-1}$ divides $i-1$, and $e_{i}=0$, otherwise. Then $K_{s}=\left\langle a_{1}, \ldots, a_{d_{s}}, t\right| t^{p^{s}}=1, t^{-1} a_{1} t=a_{d_{s}}^{-1}, t^{-1} a_{i} t=a_{i-1} a_{d_{s}}^{-e_{i}}(1<i \leq$ $\left.\left.d_{s}\right),\left[a_{i}, a_{j}\right]=1\left(1 \leq j<i \leq d_{s}\right)\right\rangle$. Furthermore, $M\left(K_{s}\right) \simeq \mathbb{Z}_{p}^{\frac{d_{s}}{2}}$, unless $p=2$ and $s=1$ in which case $M\left(K_{s}\right)=1$.

We may use the above arguments in order to note that $K_{s}$ is a metabelian group with $h\left(K_{s}\right)=d_{s}$, $\left[K_{s}, K_{s}\right] \simeq \mathbb{Z}_{p}^{d_{s}}, K_{s}^{a b}=C_{p^{s}} \times C_{p^{s}}$ and $Z\left(K_{s}\right)=\{1\}$. However, $K_{s} \notin \mathfrak{P}$, but $K_{s} \in \mathfrak{S}$.

B. Eick and W. Nickel 8 have studied the nonabelian tensor square of $G_{n}$, when $n=p$. For $p=2$ we have the infinite dihedral group $G_{2}=D_{\infty}=\left\langle a, x \mid a^{x}=a^{-1}, x^{2}=1\right\rangle=C_{2} \ltimes \mathbb{Z}$. Quoting [8, Figure at p.943], the following list holds:

$$
h\left(G_{2} \otimes G_{2}\right)=h\left(G_{2}\right)=1, h\left(G_{3} \otimes G_{3}\right)-h\left(G_{3}\right)=3-2=1,
$$




$$
h\left(G_{5} \otimes G_{5}\right)-h\left(G_{5}\right)=6-4=2, h\left(G_{7} \otimes G_{7}\right)-h\left(G_{7}\right)=9-6=3, \ldots
$$

With the help of GAP [20] one can see that the same list is true when $s=1, p=2,3,5,7$ and we deal with $K_{2}=C_{2} \ltimes \mathbb{Z}_{2}, K_{3}=C_{3} \ltimes \mathbb{Z}_{3}^{2}, K_{5}=C_{5} \ltimes \mathbb{Z}_{5}^{4}, K_{7}=C_{7} \ltimes \mathbb{Z}_{7}^{6}$. Then it would be interesting to detect the properties of the following function from the set of the integers onto the set of the integers

$$
f: h(S) \in\{h(S) \mid S \in \mathfrak{S}\} \mapsto f(h(S))=h(S \otimes S)-h(S) .
$$

Remark 2.4. I. Nakaoka and M. Visscher show that $S \otimes S \in \mathfrak{S}$, whenever $S \in \mathfrak{S}$ (see [4, 16, 21]) and so $f$ is well-posed. On another hand, G. Ellis [10] and P. Moravec [16] show that $F \otimes F \in \mathfrak{F} \cap \mathfrak{P}$, whenever $F \in \mathfrak{F} \cap \mathfrak{P}$. Then $0=h\left(C_{2}\right) \mapsto f(0)=0$, or more generally, $0=h(F) \mapsto f(0)=0$ for all $F \in \mathfrak{F} \cap \mathfrak{P}$, but also $1=h\left(G_{2}\right) \mapsto f(1)=0$. Hence $f$ is not injective. In fact $N(f)=$ $\{h(S) \mid f(h(S))=0\}=\{h(S \otimes S)=h(S) \mid S \in \mathfrak{S}\}$. Finally, one can note that $f$ is neither additive nor multiplicative.

The next property of the Hirsch length is well-known.

Lemma 2.5 (See [14, §1.3). If $A, B \in \mathfrak{S}$ and $\varphi: A \rightarrow B$ is a homomorphism of groups, then $h(A)=h(\varphi(A))+h(\operatorname{ker} \varphi)$. In particular, the Hirsch length is additive on the extensions.

We have immediately the next consequence.

Corollary 2.6. $f(h(S)) \leq h\left(J_{2}(S)\right)$ for all $S \in \mathfrak{S}$.

Proof. (1.2) shows that $S \otimes S \in \mathfrak{S}$ is a central extension of $J_{2}(S)$ by $[S, S]$. From Lemma 2.5] $h(S \otimes S)=h\left(J_{2}(S)\right)+h([S, S])$. On another hand, $[S, S] \leq S$ implies $h([S, S]) \leq h(S)$ and so $h(S \otimes S) \leq h\left(J_{2}(S)\right)+h(S)$ from which the result follows.

We recall the following information on the structure of $J_{2}(G), \nabla(G)$ and $G \otimes G$.

Proposition 2.7 (See [3], Corollary 1.4). Let $G$ be a group such that $G^{a b}$ is abelian finitely generated with no elements of square order. Then $J_{2}(G)=\Gamma\left(G^{a b}\right) \times M(G)$.

Proposition 2.8 (See [3], Theorem 1.3 (iii)). Let $G$ be a group such that either $G^{a b}$ has no elements of square order or $G^{\prime}$ has a complement in $G$. Then $\nabla(G) \simeq \nabla\left(G^{a b}\right)$ and $G \otimes G \simeq \nabla(G) \times(G \wedge G)$.

The linear growth of (2.12) is described by the next result.

Proposition 2.9. In Lemma 2.3 let $s=1, p \neq 2$ and $K_{p}=C_{p} \ltimes \mathbb{Z}_{p}^{p-1}$ be the corresponding pro-p-group. Then $f\left(h\left(K_{p}\right)\right)=\frac{1}{2}(p-1)$. In particular, $f\left(h\left(K_{p}\right)\right)=h\left(J_{2}\left(K_{p}\right)\right)=h\left(M\left(K_{p}\right)\right)$ has a linear growth.

Proof. We claim that (1.2) is equivalent to the following diagram

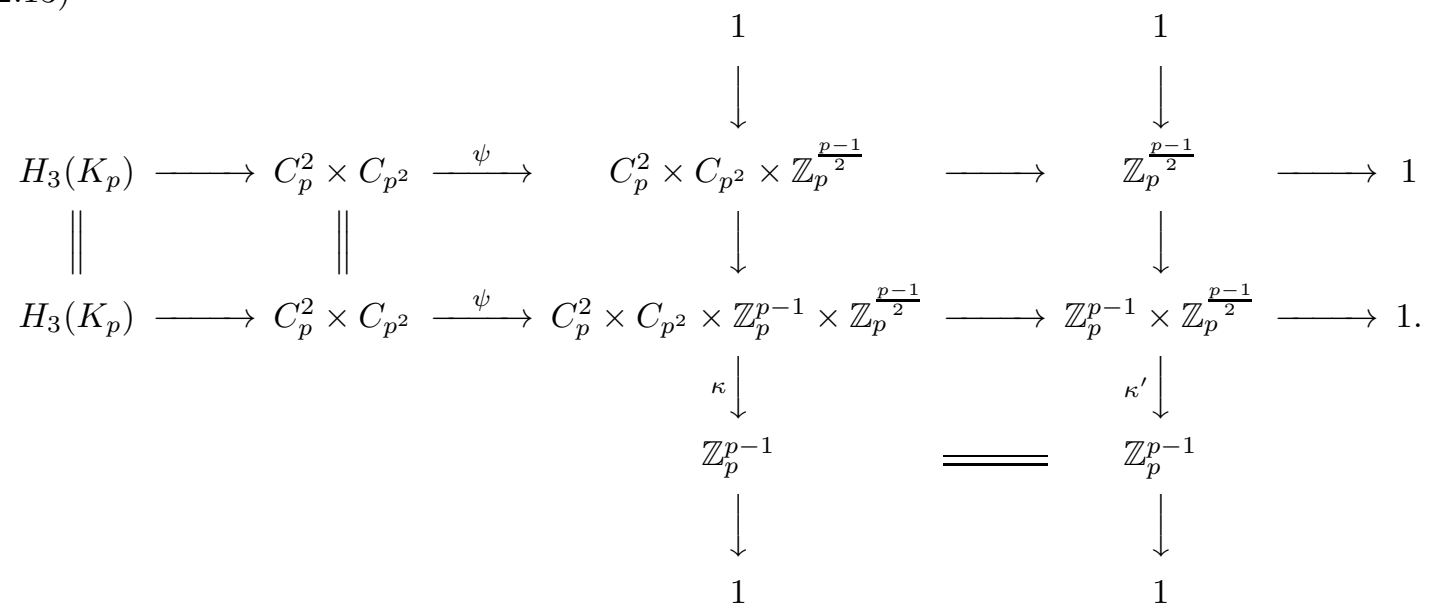

From [5, §2, (13), p.181],

$$
\Gamma\left(K_{p}^{a b}\right)=\Gamma\left(C_{p} \times C_{p}\right)=C_{p} \times C_{p} \times\left(C_{p} \otimes_{\mathbb{Z}} C_{p}\right)=C_{p} \times C_{p} \times C_{p^{2}} .
$$


Note that $C_{p} \otimes_{\mathbb{Z}} C_{p}=C_{p^{2}}$ is an elementary fact on the usual abelian tensor product. Still by [5. $\S 2]$,

$$
\psi\left(\Gamma\left(C_{p} \times C_{p}\right)\right)=\nabla\left(K_{p}\right)=C_{p} \times C_{p} \times C_{p^{2}}
$$

From Lemma 2.3. $M\left(K_{p}\right)=\mathbb{Z}_{p}^{\frac{p-1}{2}}$. We do not have elements of square order in $K_{p}^{a b}=C_{p} \times C_{p}$ and Proposition 2.7 yields $J_{2}\left(K_{p}\right) \simeq \Gamma\left(K_{p}^{a b}\right) \times M\left(K_{p}\right) \simeq C_{p} \times C_{p} \times C_{p^{2}} \times \mathbb{Z}_{p}^{\frac{p-1}{2}}$.

The commutativity of (1.2) shows that $K_{p} \wedge K_{p}$ is a central extension of $M\left(K_{p}\right)=$ ker $\kappa^{\prime}$ by $\left[K_{p}, K_{p}\right]$, which are both normal abelian subgroups of $K_{p} \wedge K_{p}$, then $K_{p} \wedge K_{p}=\left\langle M\left(K_{p}\right),\left[K_{p}, K_{p}\right]\right\rangle=$ $M\left(K_{p}\right)\left[K_{p}, K_{p}\right]=\left\langle\mathbb{Z}_{p}^{p-1}, \mathbb{Z}_{p^{2}}^{\frac{p-1}{2}}\right\rangle=\mathbb{Z}_{p}^{p-1} \mathbb{Z}_{p}^{\frac{p-1}{2}}$. On another hand,

$$
\left[M\left(K_{p}\right), M\left(K_{p}\right)\right]=\left[\left[K_{p}, K_{p}\right],\left[K_{p}, K_{p}\right]\right]=1
$$

implies

$$
\left[K_{p} \wedge K_{p}, K_{p} \wedge K_{p}\right]=\left[M\left(K_{p}\right)\left[K_{p}, K_{p}\right], M\left(K_{p}\right)\left[K_{p}, K_{p}\right]\right]=\left[M\left(K_{p}\right), M\left(K_{p}\right)\right]\left[M\left(K_{p}\right),\left[K_{p}, K_{p}\right]\right]
$$

$$
\left[\left[K_{p}, K_{p}\right],\left[K_{p}, K_{p}\right]\right]\left[M\left(K_{p}\right),\left[K_{p}, K_{p}\right]\right]=\left[M\left(K_{p}\right),\left[K_{p}, K_{p}\right]\right]
$$

Since $M\left(K_{p}\right)=\mathbb{Z}_{p}^{\frac{p-1}{2}} \leq \mathbb{Z}_{p}^{p-1}=\left[K_{p}, K_{p}\right]$, we deduce $C_{K_{p} \wedge K_{p}}\left(\left[K_{p}, K_{p}\right]\right) \leq C_{K_{p} \wedge K_{p}}\left(M\left(K_{p}\right)\right)$ and then

$$
\left[K_{p}, K_{p}\right] \leq C_{K_{p} \wedge K_{p}}\left(\left[K_{p}, K_{p}\right]\right) \leq C_{K_{p} \wedge K_{p}}\left(M\left(K_{p}\right)\right)
$$

which implies $\left[M\left(K_{p}\right),\left[K_{p}, K_{p}\right]\right]=1$. We conclude that $K_{p} \wedge K_{p}$ is abelian and then the central extension is actually a direct product of the form $K_{p} \wedge K_{p}=\mathbb{Z}_{p}^{p-1} \times \mathbb{Z}_{p}^{\frac{p-1}{2}}$. From Proposition 2.8

$$
K_{p} \otimes K_{p}=C_{p} \times C_{p} \times C_{p^{2}} \times \mathbb{Z}_{p}^{p-1} \times \mathbb{Z}_{p^{\frac{p-1}{2}}}
$$

Then

$$
h\left(M\left(K_{p}\right)\right)=h\left(J_{2}\left(K_{p}\right) / \nabla\left(K_{p}\right)\right)=h\left(J_{2}\left(K_{p}\right)\right)=\frac{p-1}{2} .
$$

We conclude from (2.13) and Lemma 2.5 that

$$
h\left(K_{p} \otimes K_{p}\right)=h\left(\kappa\left(K_{p} \otimes K_{p}\right)\right)+h\left(J_{2}\left(K_{p}\right)\right)=(p-1)+h\left(M\left(K_{p}\right)\right)=\frac{3}{2}(p-1) .
$$

Therefore $f\left(h\left(K_{p}\right)\right)=h\left(J_{2}\left(K_{p}\right)\right)=\frac{1}{2}(p-1)$.

The methods in the above proof continue to be valid when $s>1$. Therefore we draw the following result, which has independent interest and, in view of [13, Theorem 7.4.12, Corollary 7.4.13], describes the nonabelian tensor square of all pro- $p$-groups of finite coclass with trivial center.

Theorem 2.10. If $s>1$ and $p$ is an odd prime, then $K_{s} \otimes K_{s}=C_{p^{s}}^{2} \times C_{p^{2 s}} \times \mathbb{Z}_{p}^{\frac{3}{2} d_{s}}$.In particular, $f\left(h\left(K_{s}\right)\right)=h\left(J_{2}\left(K_{s}\right)\right)=h\left(M\left(K_{s}\right)\right)$ has a linear growth.

Proof. Mutatis mutandis, we may argue as in the proof of Proposition 2.9.

The computational data show that $M\left(G_{p}\right)=\mathbb{Z}^{\frac{p-1}{2}}$. In alternative, an argument as in $[9$, Proof of Theorem 7] can be applied, that is, we may express the Schur's Formula for $M\left(G_{p}\right)$, beginning from the presentation in Corollary 2.6. Equivalently, we may work via duality, since the cohomology of $G_{p}$ is known by [1]. This justifies the assumption of the next result.

Corollary 2.11. Assume $M\left(G_{p}\right)=\mathbb{Z}^{\frac{p-1}{2}}$ for all primes $p \neq 2$. Then $f\left(h\left(G_{p}\right)\right)=\frac{1}{2}(p-1)$. In particular, $f\left(h\left(G_{p}\right)\right)=h\left(J_{2}\left(G_{p}\right)\right)=h\left(M\left(G_{p}\right)\right)$ has a linear growth.

Proof. We may argue as in the proof of Proposition 2.9, replacing $K_{p}$ with $G_{p}$. 
The above results prove that there are crystallographic groups of holonomy $p \neq 2$ which achieve the bound in Corollary 2.6. The same is true for the pro- $p$-group $K_{p}$ with $p \neq 2$. Note that Proposition 2.9 describes rigorously the structure of $K_{p} \otimes K_{p}$ with respect to that of $K_{p}$ in terms of their torsion-free factors. The same is true for $G_{p}$ by Corollary 2.11, The fact that (2.12) has a linear growth can be translated in terms of restrictions on the Schur multiplier as follows.

Corollary 2.12. If $f(h(S))=c h(S)$ for some integer $c \geq 0$ and $S \in \mathfrak{S}$, then $h(M(S)) \leq$ $h(S)^{2}+(c+1) h(S)$. The equality holds, whenever $S \in \mathfrak{F}$.

Proof. We have $f(h(S))=h(S \otimes S)-h(S)=\left(h\left(J_{2}(S)\right)+h([S, S])\right)-h(S)=h(M(S))-h(\nabla(S))+$ $h([S, S])-h(S)$. Now we may always write $s \otimes s=(s \otimes 1)(1 \otimes s)$ in a unique way and then the $\operatorname{map} \iota: s \otimes s \in \nabla(S) \mapsto \iota(s \otimes s)=\iota((s \otimes 1)(1 \otimes s))=\iota(s \otimes 1) \iota(1 \otimes s)=(s, s) \in S \times S$ is a monomorphism. Therefore $h(\nabla(S)) \leq h(S)^{2}$ and so $h(M(S))=f(h(S))+h(\nabla(S))-h([S, S])+$ $h(S) \leq f(h(S))+h(\nabla(S))+h(S) \leq c h(S)+h(S)^{2}+h(S)$ from which the result follows.

Unfortunately, (2.12) has not a linear growth for all groups in $\mathfrak{S}$ and we cannot predict its form in general. Already in $\mathfrak{P}$ there are examples in this sense (see [8, Figure at p.943]). However, a nice circumstance is described below.

Corollary 2.13. There exists a metabelian group $G$ with trivial center for which $f(h(G))=$ $h(M(G))=\frac{1}{2} p^{s-1}(p-1)$, where $s>1$ and $p$ is an odd prime.

Proof. Consider $G=K_{s}$. By Lemma 2.3, $h\left(M\left(K_{s}\right)\right)=\frac{1}{2} p^{s-1}(p-1)$. From Theorem [2.10, $f\left(h\left(K_{s}\right)\right)=h\left(K_{s} \otimes K_{s}\right)-h\left(K_{s}\right)=\left(p^{s-1}(p-1)+\frac{1}{2} p^{s-1}(p-1)\right)-p^{s-1}(p-1)=\frac{1}{2} p^{s-1}(p-1)=$ $h\left(M\left(K_{s}\right)\right)$.

We end the section with an explicit description for (2.12), modifying a classic argument of N. Rocco, which can be found in [3, Theorem 1] (see also [3, Observation]).

Theorem 2.14. Let $G$ be a group in $\mathfrak{P}$ such that $G^{a b}=\prod_{i=1}^{n} C_{p^{e_{i}}}$, for integers $1 \leq e_{i} \leq e_{j}$ such that $1 \leq i<j \leq n, p$ odd prime and $d=\sum_{i=1}^{n}(n-i) e_{i}$.

(a) If $G$ is finite, then $|G \otimes G|=p^{d}|G||M(G)|$.

(b) If $G$ is infinite, then $f(h(G))=h(M(G))$.

Proof. (a). Assume $G$ is finite. Since $G^{a b}$ is finitely generated and has no elements of order two, all the hypotheses of [3, Theorem 1] are satisfied and so $G \otimes G \simeq \nabla(G) \times G \wedge G$. From this and (1.2) we deduce

$$
|G \otimes G|=\frac{|\nabla(G)|}{\left|G^{a b}\right|}|G||M(G)|=\frac{\left|\Gamma\left(G^{a b}\right)\right|}{\left|G^{a b}\right|}|G||M(G)|=\left|\prod_{i=1}^{n}\left(C_{p^{e_{i}}}\right)^{n-i}\right||G||M(G)|=p^{d}|G||M(G)|
$$

where $d=\sum_{i=1}^{n}(n-i) e_{i}$.

(b). Assume $G$ is infinite. From Proposition 2.7 and Lemma 2.5 we conclude that $h\left(J_{2}(G)\right)=$ $h\left(\Gamma\left(G^{a b}\right)\right)+h(M(G))=h(M(G))$, where the last equality is due to the fact that $\Gamma\left(G^{a b}\right)$ is periodic. Proceeding as in (2.22),

$$
h(G \otimes G)=h(\kappa(G \otimes G))+h([G, G])=h\left(J_{2}(G)\right)+h([G, G])=h(M(G))+h([G, G]) .
$$

Subtracting $h(G)$, we find$$
f(h(G))=h(G \otimes G)-h(G)=h(M(G))-(h(G)-h([G, G]))=h(M(G))-h\left(G^{a b}\right)=h(M(G)),
$$

since $G^{a b}$ is periodic.

Remark 2.15. It is not used the hypothesis $G \in \mathfrak{P}$ in Theorem 2.14 (a) and so this part of the result is true for an arbitrary finite group. 


\section{SOME EVIDENCES}

The present section is devoted to evaluate (2.12) for other classes of groups for which it is known their nonabelian tensor product. A Bieberbach group $B$ is an extension of a free abelian group $L$ (called lattice group) of finite rank by a group $P$ (called holonomy group). Following the notation of Lemma 2.1. we are fixing $A=L, B=\Gamma$ and $Q=P$, imposing a precise choice for these groups. The dimension of $B$ is the rank of $L$. It is easy to see that $G_{p}$, studied in the previous section, is of this form, once $L=\mathbb{Z}^{n-1}$ and $P=C_{n}$. It is known that

$$
B_{1}(2)=\left\langle a, x, y \mid a^{2}=y, a x a^{-1}=x^{-1},[a, y]=[x, y]=1\right\rangle
$$

is a Bieberbach group of dimension 2 with point group $C_{2}$ and that the groups

$$
B_{1}(n)=B_{1}(2) \times \mathbb{Z}^{n-2} \text { for } n \geq 2
$$

are Bieberbach groups of dimension $n$ with point group $C_{2}$. More details can be found in [15]. The next two results check (2.12) on $B_{1}(2)$ and $B_{1}(n)$.

Corollary 3.1. In $B_{1}(2)$ we have that $f$ is constant to 0 .

Proof. From [15, Theorem 4.1] we have

$$
B_{1}(2) \otimes B_{1}(2)=C_{2} \times C_{4} \times \mathbb{Z}^{2} .
$$

Still from [15] we know that $M\left(B_{1}(2)\right)$ is trivial. Now $f\left(h\left(B_{1}(2)\right)\right)=h\left(B_{1}(2) \otimes B_{1}(2)\right)-h\left(B_{1}(2)\right)=$ $2-2=0$ and the result follows

Corollary 3.2. In $B_{1}(n)$ we have that $f\left(h\left(B_{1}(n)\right)\right)=n^{2}-3 n+4$ for all $n>2$.

Proof. From [15, Corollary 4.1] we have

$$
B_{1}(n) \otimes B_{1}(n)=C_{2}^{2 n-3} \times C_{4} \times \mathbb{Z}^{(n-1)^{2}+1} .
$$

Still from [15] we know that $M\left(B_{1}(2)\right)=n-2$ and so it is nontrivial. Now $f\left(h\left(B_{1}(n)\right)\right)=$ $h\left(B_{1}(n) \otimes B_{1}(n)\right)-h\left(B_{1}(n)\right)=\left((n-1)^{2}+1\right)-(n-2)=n^{2}-2 n+2-n+2=n^{2}-3 n+4$ and the result follows.

In a certain sense Theorem 2.14 (b) forces the growth of (2.12) to be equal to that of the Schur multiplier, when the abelianization of the group is the direct product of finite cyclic groups. Is this condition really necessary? Unfortunately, the answer is positive and $B_{1}(n)$ for $n>2$ shows it.

Corollary 3.3. For all $n>2, f\left(h\left(B_{1}(n)\right)\right)$ has not a linear growth but $h\left(M\left(B_{1}(n)\right)\right)$ has a linear growth.

Proof. $f\left(h\left(B_{1}(n)\right)\right)=n^{2}-3 n+4$ and $h\left(M\left(B_{1}(n)\right)\right)=n-2$.

Recent progresses in 3, 4, show that the nonabelian tensor product of Bieberbach groups has a similar structure with respect to that of the free solvable groups of finite rank and free nilpotent groups of finite rank. Therefore we have the following results.

Corollary 3.4. Let $F$ be the free group of finite rank $r \geq 1$ and $G=F / F^{(d)}$ be the free solvable group of derived length $d \geq 1$ and rank $r$. If $F^{\prime}$ is periodic, then $f(h(G)) \leq \frac{1}{2} r(r-1)$. In particular, if $h(G)=r$, then the equality holds and $f(h(G))=\frac{1}{2} r(r-1)$.

Proof. We may apply 3, Corollary 2.4] and so $G \otimes G=\mathbb{Z}^{\frac{1}{2} r(r+1)} \times F^{\prime} /\left[F, F^{(d)}\right]$. Lemma 2.5 implies $h(G \otimes G)=\frac{1}{2} r(r+1)$. Of course $h(G) \leq r$. Then $f(h(G)) \leq \frac{1}{2} r(r+1)-r=\frac{1}{2} r(r-1)$, as claimed.

Corollary 3.5. Let $G$ be the free nilpotent group of rank $r \geq 1$ and class $c \geq 1$. If $G^{\prime}$ is periodic, then $f(h(G)) \leq \frac{1}{2} r(r-1)$. In particular, if $h(G)=r$, then the equality holds and $f(h(G))=$ $\frac{1}{2} r(r+1)$.

Proof. Note that nilpotent groups are solvable and so it is meaningful to consider $f(h(G))$. Applying [3. Corollary 2.3], $G \otimes G=\mathbb{Z}^{\frac{1}{2} r(r+1)} \times G^{\prime}$ and the remainder is similar to the previous corollary. 
A. ERFANIAN, F. G. RUSSO, AND N.H. SARMIN

However, Lemma 2.1 imposes the following question, which we leave open in its generality.

Open Question 3.6. What is the growth of $h(\Gamma \otimes \Gamma)$ with respect to $h(\Gamma)$, where $\Gamma$ is an arbitrary extension of two abelian groups $A$ and $Q$ as in Lemma 2.1?

\section{REFERENCES}

[1] A. Adem, J. Ge, J. Pan and N. Petrosyan, Compatible actions and cohomology of crystallographic groups, J. Algebra 320 (2008), 341-353.

[2] J.R. Beuerle, L.-C. Kappe, Infinite metacyclic groups and their non-abelian tensor squares, Proc. Edinb. Math. Soc. 43 (2000) 651-662.

[3] R. Blyth, F. Fumagalli and M. Morigi, Some structural results on the non-abelian tensor square of groups, Preprint, Cornell University, arXiv:0810.4620 2008.

[4] R. Blyth and R. Morse, Computing the nonabelian tensor squares of polycyclic groups, J. Algebra 321 (2009), 2139-2148.

[5] R. Brown, D.L. Johnson and E. F. Robertson, Some computations of non-abelian tensor products of groups, J. Algebra 111 (1987), 177-202.

[6] R. Brown and J.-L. Loday, Van Kampen theorems for diagrams of spaces, Topology 26 (1987), 311-335.

[7] K. Dennis, In search of new homology functors having a close relationship to K-theory, Preprint, Cornell University, 1976.

[8] B. Eick and W. Nickel, Computing the Schur multiplicator and the nonabelian tensor square of a polycyclic group, J. Algebra 320 (2008), 927-944.

[9] B. Eick, Schur multiplicators of infinite pro-p-groups with finite coclass, Israel J. Math. 166 (2008), 147-156.

[10] G. Ellis, The nonabelian tensor product of finite groups is finite, J. Algebra 111 (1987), 203-205.

[11] G. Ellis, Tensor products and q-crossed modules, J. London Math. Soc. 2 (1995), 241-258.

[12] L.-C. Kappe, N.H. Sarmin and M.P. Visscher, Two-generator two-groups of class two and their nonabelian tensor squares, Glasgow Math. J. 41 (1999), 417-430.

[13] C. R. Leedham-Green and S. McKay, The Structure of Groups of Prime Power Order, Oxford University Press, Oxford, 2002.

[14] J.C. Lennox and D.J.S. Robinson, The Theory of Infinite Soluble Groups, Oxford Univerisity Press, Oxford, 2004.

[15] R. Masri, The nonabelian tensor squares of certain Bieberbach groups with cyclic point group of order 2, Phd thesis, Universiti Teknologi Malaysia, 2009.

[16] P. Moravec, The nonabelian tensor product of polycyclic groups is polycyclic, J. Group Theory 10 (2007), 795-798.

[17] R. F. Morse, Private communication, 2009.

[18] D. Ramras, Quillen-Lichtenbaum phenomena in the stable representation theory of crystallographic groups, Cornell University Library, Arxiv: 1007.0406, 2010.

[19] N.H. Sarmin, Infinite two generator groups of class two and their non-abelian tensor squares, Int. J. Math. Math. Sci. 32 (10) (2002), 615-625.

[20] The GAP Group, GAP-Groups, Algorithms and Programming, version 4.4, available at http://ww.gap-system.org, 2005.

[21] M.P. Visscher, On the nilpotency class and solvability length of nonabelian tensor products of groups, Arch. Math. (Basel) 73 (1999), 161-171.

Acknowledgment. The first and the second author are grateful to the Department of Mathematics and the Ibnu Sina Institute of the Universiti Teknologi Malaysia for the hospitality in the summer of 2009, when the initial part of this manuscript was written. We also thank Prof. B. Eick, who suggested [8, 9, Prof. R. F. Morse and Dr. P. Moravec, who communicated some inaccuracies in the original version of the present paper. Finally, we appreciated some email contributions of R. Brown, A. Caranti, D. Feirtenschlager, R. Hartung, M. Horn and D. Ramras in 2010.

Mathematics Department and, Centre of Excellence in Analysis on Algebraic Structures, Ferdowsi University of Mashhad, P.O.Box 1159, 91775, Mashhad, Iran

E-mail address: erfanian@math.um.ac.ir

Laboratorio di Dinamica e Geotecnica - Strega, Universitá del Molise, via Duca degli Abruzzi, Termoli (CB)

E-mail address: francescog.russo@yahoo.com

Department of Mathematics, Faculty of Science, Universiti Teknologi Malaysia, 81310 Utm Johor Bahru, Johor, Malaysia

E-mail address: nhs@utm.my 